\title{
Relações entre Estresse, Distorções Cognitivas e Otimismo em Pacientes com Hipertensão
}

\author{
Diego dos Santos Gonçalves Pacheco ${ }^{1}$ \\ ${ }^{1}$ Policlínica Naval de Campo Grande, RJ, Brasil
}

\author{
Lucia Emmanoel Novaes Malagris ${ }^{2}$ \\ ${ }^{2}$ Universidade Federal do Rio de Janeiro, RJ, Brasil
}

Resumo: O estudo teve como objetivo investigar possíveis relações entre hipertensão, estresse, otimismo e distorções cognitivas. Foram utilizados como instrumentos um questionário sociobiodemográfico, o Inventário de Sintomas de Stress para Adultos de Lipp, o Questionário de Distorções Cognitivas e o Teste de Orientação de Vida Revisado. A amostra foi constituída de cinquenta pessoas com hipertensão e cinquenta sem hipertensão, de 42 a 69 anos. A maioria dos participantes não estava estressada (67\%), e não houve diferença entre os grupos quanto à presença de estresse, grau de otimismo ou distorções cognitivas. No grupo com hipertensão, a presença de estresse relacionou-se de maneira direta às distorções cognitivas, sem relacionarse ao otimismo. Já no grupo sem hipertensão a presença de estresse relacionou-se de maneira inversa ao otimismo, mas não com o escore geral de distorções cognitivas. Os resultados apontaram que a presença de hipertensão pode atuar sobre as relações entre o estresse, distorções cognitivas e otimismo.

Palavras-chave: Estresse, Hipertensão, Otimismo, Processos Cognitivos.

\section{Relation between Stress, Cognitive Distortions and Optimism in Patients with Hypertension}

\begin{abstract}
This study investigated possible relations between hypertension, stress, optimism and cognitive distortions. The instruments used were a sociobiodemographic questionnaire, Lipp's Inventory of Stress Symptoms for Adults, the Cognitive Distortion Questionnaire and the Life Orientation Test. The sample consisted of 50 people with hypertension and 50 without ranging from 42 to 69 years. Most people were not stressed (67\%), and there was no significant difference between groups regarding the presence of stress, level of optimism or cognitive distortions. In the hypertension group, the presence of stress was significant and directly related to cognitive distortions, not relating to optimism. In the group without hypertension, the presence of stress was inversely related to optimism, but there was no relation to the general score of cognitive distortions. The results showed that the presence of hypertension can act upon the relations between stress, cognitive distortions and optimism.
\end{abstract}

Keywords: Stress, Hypertension, Optimism, Cognitive Processes. 


\title{
Relaciones entre Estrés, Distorsiones Cognitivas y Optimismo en Pacientes con Hipertensión
}

\begin{abstract}
Resumen: El estudio pretende investigar posibles relaciones entre hipertensión, estrés, optimismo y distorsiones cognitivas. Se usaron como instrumentos un cuestionario sociobiodemográfico, el Inventario de Síntomas de Estrés para Adultos de Lipp, el Cuestionario de Distorsiones Cognitivas y el Test de Orientación Vital Revisado. La muestra estuvo compuesta de 50 personas con hipertensión y 50 sin hipertensión, de edades entre 42 y 69 años. La mayoría de los participantes no estaban estresados $(67 \%)$, y no hubo diferencias entre los grupos en cuanto a la presencia de estrés, grado de optimismo o distorsiones cognitivas. En el grupo con hipertensión, la presencia de estrés se relacionó de manera directa a las distorsiones cognitivas, sin relacionarse con el optimismo. En el grupo sin hipertensión, la presencia de estrés se relacionó de manera inversa al grado de optimismo, pero no con el puntaje general de distorsiones cognitivas. Los resultados apuntaron que la presencia de hipertensión puede actuar sobre las relaciones entre el estrés, las distorsiones cognitivas y el optimismo.
\end{abstract}

Palabras clave: Estrés, Hipertensión, Optimismo, Procesos Cognitivos.

\section{Introdução}

Segundo dados da Vigilância de Fatores de Risco e Proteção para Doenças Crônicas por Inquérito Telefônico de2013 (Brasil,2014), cerca de24\% dos adultos brasileiros apresentam hipertensão arterial (HA), com evidente tendência de aumento com a idade, sendo uma das causas de maior redução de qualidade e expectativa de vida.

As Sociedades Brasileiras de Cardiologia, Hipertensão e Nefrologia (2010) afirmam que "hipertensão arterial sistêmica é uma condição clínica multifatorial caracterizada por níveis elevados e sustentados de pressão arterial (PA)" (p. 1). Advém de uma relação complexa, não totalmente compreendida, entre o ambiente, o sistema nervoso autônomo e os sistemas cardiovascular e renal, associados à predisposição genética. Neste sentido, é reconhecido que os estímulos psicoemocionais influenciam a fisiopatogenia da doença, mas a força de suas influências, tanto na origem quanto na consequência para pessoas sabidamente com hipertensão, ainda é desconhecida (Cade, 2001). Um dos fatores psicológicos mais reconhecidamente associados ao quadro é o estresse, sendo seu controle recomendado pelas VI Diretrizes Brasileiras de Hipertensão Arterial (Sociedade Brasileira de Cardiologia, Sociedade Brasileira de Hipertensão, \& Sociedade Brasileira de Nefrologia, 2010).
Lipp e Malagris (2011) definem estresse como uma reação física e psicológica do organismo ao lidar com determinada situação que provoque alterações emocionais como irritação, medo ou grande felicidade. Além disto, afirmam que a força desta reação sobre o organismo depende da capacidade deste em vencer ou se adaptar ao estressor, buscando retornar ao equilíbrio de funcionamento típico da homeostase.

De acordo com o modelo quadrifásico de estresse, podemos identificar quatro etapas distintas deste fenômeno (Lipp \& Malagris, 2011): a) alerta, primeira reação do organismo frente ao estímulo estressor, preparando o corpo para um enfrentamento agudo; b) resistência, quando as defesas são preparadas para um estressor de maior duração, e o organismo tenta se acomodar ao evento para retomar a homeostase; c) quase-exaustão, quando não consegue mais adaptar-se ou resistir de maneira eficaz à origem do estresse, embora continue na luta pela homeostase; d) exaustão, quando o organismo exaure sua capacidade de lidar com o problema, reaparecendo sintomas do estágio de alerta, mas em maior intensidade, e outros que denunciam que o organismo está sendo vencido pelo estresse.

Para Margis (2003), a resposta ao stress é composta por elementos únicos de predisposições e aprendizagens, em uma relação com as demandas do meio. $\mathrm{O}$ indivíduo conta, portanto, com a ativação de 
um sistema biológico inato, mas também com uma série de crenças que funcionam como formas de proteção ou fatores de risco sobre a adaptação ao stress gerado. O desenvolvimento do processo de estresse depende, em parte, da avaliação que o indivíduo tem sobre o estressor e sua própria capacidade em lidar com ele. Uma pessoa que considera um estímulo como excessivamente perigoso ou que se considera exageradamente frágil terá provavelmente uma resposta mais intensa de estresse do que uma pessoa com avaliações mais realistas (Rangé \& Masci, 2001). As distorções cognitivas, portanto, entendidas como interpretações tendenciosas da realidade que trazem sofrimento ao indivíduo (Beck, 2013), podem ter importante papel na origem e manutenção do estresse, e seu tratamento é um dos principais objetivos das intervenções específicas para sua administração (Rangé \& Masci, 2001).

Muitos pesquisadores vêm se dedicando ao estudo de intervenções para o estresse no contexto de pessoas com HA, seja por meio do relaxamento como exclusividade de tratamento (Malagris, Lip, \& Chicayban, 2014; Jose \& D’Almeida, 2013) ou de adaptações de estratégias cognitivo-comportamentais, que se aprimoram continuamente (Blom et al., 2014; Lipp, 2000, Malagris et al., 2009). Somando-se a isto, com o advento da psicologia positiva, uma significativa parcela das pesquisas passou a olhar para os fatores que favorecem a resiliência dos indivíduos (Schrank, Brownell, Tylee, \& Slade, 2014). Nesse sentido, além de emoções positivas e locus de controle interno, uma característica que tem chamado atenção por sua relação com o estresse é o nível de otimismo (Seligman, 2011) que, pela definição de Carver, Scheier e Segerstrom (2010), pode ser entendido como a tendência, de maneira estável, que uma pessoa tem em crer que terá mais resultados positivos do que negativos. Algumas pesquisas apontam que o otimismo age como um fator de proteção ao estresse em diversos contextos, seja em atletas de competição (Gustafsson \& Skoog, 2012), militares em locais de combate (Thomas, Britt, Odle-Dusseauet, \& Bliese, 2011) ou enfermeiras hospitalares (Chang \& Chan, 2015). As explicações mais comuns para este fenômeno dizem respeito a pessoas otimistas tenderem a apresentar estratégias de enfrentamento mais adaptativas (Scheier \& Carver, 1992) e busca de suporte social (Puig-Perez et al., 2015). Cabe destacar, entretanto, que o otimismo, quando desproporcional e irrealista, tende a ser prejudicial, pois está associado a menores preocupações com a saúde e, consequentemente, a maior comportamento de risco (Reyes-Velázquez \& Sealey-Potts, 2015).

Considerando estudos no campo da Psicologia da Saúde e da psicologia positiva, o presente estudo investigou de que forma o estresse, as distorções cognitivas e o otimismo se relacionam com a hipertensão, por meio de uma comparação entre um grupo de pessoas com a doença e outro que não a apresentava.

\section{Método}

$\mathrm{O}$ estudo se constituiu em uma pesquisa quantitativa, de levantamento e exploratória, na qual pretendeu-se compreender algumas relações entre estresse, otimismo e distorções cognitivas em pacientes com hipertensão, comparando-os com pessoas sem hipertensão.

\section{Participantes}

Fizeram parte do estudo cem participantes, divididos da seguinte maneira: cinquenta com diagnóstico de HA (Grupo A) e cinquenta sem HA (Grupo B). O Grupo A se constituiu de usuários do Sistema de Saúde da Marinha, sendo composto por militares ou dependentes de militares da Marinha do Brasil diagnosticados com HA por um profissional de saúde. Os participantes do Grupo B, por sua vez, eram militares, dependentes, ou acompanhantes sem qualquer vínculo com a Marinha do Brasil.

Os critérios de inclusão na pesquisa para o Grupo A foram: a) apresentar HA diagnosticada por, no mínimo, um ano; b) ter cursado e concluído, no mínimo, o sexto ano do ensino fundamental; c) não apresentar transtornos mentais atuais ou doenças crônicas, de acordo com relato próprio e, posteriormente, consulta ao prontuário médico; d) não estar em acompanhamento psicológico.

Já os participantes do Grupo B foram selecionados de modo pareado às informações etárias e de sexo obtidas pelo Questionário Sociobiodemográfico aplicado ao Grupo A. Além disto, não possuíam diagnóstico de HA e tinham que atender aos critérios de inclusão já descritos. O pareamento de idade entre os Grupos A e B permitiu diferença de até cinco anos, por entender que esta variação não comprometeria os resultados na comparação entre os grupos. 


\section{Materiais e instrumentos}

Quanto aos materiais e instrumentos, além do Termo de Consentimento Livre e Esclarecido (TCLE), foram utilizados:

- Prontuário - Tem o objetivo de registrar os dados pessoais e de atendimentos do paciente na instituição.

- Questionário Sociobiodemográfico - Tem o objetivo de obter informações para conhecimento do perfil dos participantes, como sexo, idade, nível educacional, doenças crônicas e presença de fatores de risco para doenças crônicas. Também visou confirmar a adequação aos critérios de inclusão e permitir a homogeneização dos grupos.

- Inventário de Sintomas de Stress para Adultos de Lipp (ISSL) - Elaborado por Lipp (2000), com coeficiente alfa de Cronbach de 0,9121, tem como objetivos verificar a presença de estresse no indivíduo, a fase na qual se encontra a partir do modelo quadrifásico e, ainda, investigar se os sintomas predominantes são físicos ou psicológicos.

- Questionário de Distorções Cognitivas (CDQuest) - Criado por De Oliveira, em 2010, com coeficiente alfa de Cronbach entre 0,83 e 0,86, tem o objetivo de avaliar distorções cognitivas por meio de quinze itens (um para cada distorção cognitiva) que devem ser respondidos em relação à frequência e intensidade na última semana. São elas: comparações injustas, questionamentos "e se", culpabilização, conclusões precipitadas, afirmações do tipo "deveria", personalização, generalização, leitura mental, abstração seletiva, ampliação/ minimização, rotulação, raciocínio emocional, desqualificação de aspectos positivos, previsão do futuro e pensamento dicotômico. Cada distorção possui uma pontuação entre 0 e 5 pontos, que, somadas, variam entre 0 e 75 pontos em avaliação global (De Oliveira et al., 2015).

- Teste de Orientação de Vida Revisado (LOT-R) Criado por Scheier, Carver e Bridges em 1994, com coeficiente alfa de Cronbach de 0,78 , tem o objetivo de avaliar o nível de otimismo a partir de 10 itens em Escala de Likert (Bastianello \& Pacico, 2014).

\section{Procedimento}

Este projeto foi apreciado pelo Comitê de Ética em Pesquisa com Seres Humanos do Centro de Filosofia e Ciências Humanas da Universidade Federal do Rio de Janeiro em 3 de fevereiro de 2016 (Protocolo número 1.402.363). O período de coleta dos dados foi de março a agosto de 2016.

Após aprovação da instituição, os profissionais de saúde que lá atendiam foram informados sobre a pesquisa e orientaram os pacientes que preenchiam o já citado perfil a procurar o setor de Psicologia, caso tivessem interesse em participar, depois de seu atendimento. Ao se apresentarem no setor de Psicologia, receberam maiores detalhes do pesquisador que, com o preenchimento do TCLE, prosseguiu com a aplicação dos instrumentos, na seguinte ordem: Questionário sociobiodemográfico, Inventário de Sintomas de Stress para Adultos de Lipp, Questionário de Distorções Cognitivas e Teste de Orientação de Vida Revisado. Os participantes que eram acompanhantes dos pacientes foram abordados diretamente pelo entrevistador.

Os questionários foram preenchidos pelos próprios participantes, em grupos de até cinco pessoas, guiados pelo entrevistador que lia, explicava e respondia eventuais dúvidas sobre os instrumentos. Após a administração dos instrumentos, o prontuário médico de cada participante foi verificado para confirmar as informações de idade, presença de HA ou outra doença crônica, transtorno psiquiátrico diagnosticado ou se estava em tratamento psicológico. Nos casos em que o prontuário demonstrou inelegibilidade do participante, os questionários foram descartados, conforme informado no TCLE.

No final do estudo, por questões éticas, os participantes de ambos os grupos identificados com presença de estresse foram convidados a ingressar em grupos de psicoterapia para administração do estresse oferecidos pela Policlínica Naval de Campo Grande (PNCG). Após utilização dos dados na pesquisa, o material da pesquisa foi armazenado na Instituição sob responsabilidade do pesquisador e ali permanecerá pelo período de cinco anos.

\section{Resultados e discussão}

Considerando a amostra como um todo, no que se refere ao sexo e escolaridade, participaram 67 homens e 33 mulheres, tendo $70 \%(n=70)$ da amostra completado o ensino médio. Tais dados são comuns 
na literatura envolvendo estudos com militares (Costa Accioly, Oliveira, \& Maia, 2007; Dantas, Brito, Rodrigues, Maciente, 2010), uma vez que a maior parte deles é composta por homens com esta escolaridade (Instituto Brasileiro de Geografia e Estatística, 2012). A predominância de pessoas casadas (37\%), em relação aos demais estados civis, provavelmente tem relação com a faixa etária predominante dos participantes do estudo (entre 50 e 59 anos), que segundo dados do IBGE (2012) concentra maior proporção de pessoas casadas do que em outras situações conjugais.

A concentração de faixa etária entre 50 e 59 anos (46\% no presente estudo) é um dado frequentemente encontrado em pesquisas que exploram a temática da HA (Oliveira-Martins, Oliveira, Gomes, Caramona, \& Cabrita, 2011; Serra et al., 2015) e diz respeito ao fato dessa condição aumentar com a idade (Sociedade Brasileira de Cardiologia et al., 2010). No que se refere a fatores de risco para doenças cardíacas informados pelos participantes, o presente estudo identificou como o mais comum o colesterol alto (30\%), o que é respaldado pela literatura (Oliveira-Martins et al., 2011). É frequente na sociedade o consumo de alimentos ricos neste nutriente e, quando consumido em excesso, pode promover o bloqueio dos vasos sanguíneos e favorecer o aumento da PA (Sociedade Brasileira de Cardiologia et al., 2010).

Quanto ao estresse, a maior parte das pessoas da amostra total não estava estressada (67\%). Dos que apresentaram estresse $(n=33)$ a maioria ficou concentrada na fase de resistência (82\%) e apresentou sintomas predominantemente psicológicos de estresse (63\%). Em comparação à literatura, a maior parte dos estudos envolvendo amostras com militares brasileiros chegou a resultados semelhantes, com predominância de pessoas sem sintomatologia de estresse (Costa et al., 2007; Dantas et al., 2010) ou com leve predominância de pessoas estressadas (Oliveira \& Bardagi, 2009). Na maior parte deles, houve predomínio de sintomas psicológicos (Costa et al., 2007; Oliveira \& Bardagi, 2009). Em todos os estudos apontados também houve predomínio da fase de resistência entre as pessoas estressadas. Segundo Lipp (2010), nesta fase, o organismo tenta resistir para manter-se equilibrado, ou seja, ainda que ocorra um desgaste que possa levar a maiores prejuízos, é um estado passível de prevenção e de controle caso o estressor deixe de estar presente ou caso o indivíduo desenvolva estratégias de controle do estresse. Cabe ressaltar que, embora as amostras das diferentes pesquisas possam ter algumas características em comum, a maneira como cada indivíduo expressa o estresse pode variar em função de suas estratégias de enfrentamento ou manejo de sintomas (Dantas et al., 2010).

Acerca do otimismo, cuja escala medida pelo LOT-R varia entre 0 e 30 pontos, o presente estudo teve média 26,2 $\pm 4,2$ (mediana $=28$ ). Não foram encontradas pesquisas sobre otimismo com amostras semelhantes, mas um estudo sobre a satisfação de vida em militares portugueses com média de idade $34,87 \pm 7,35$ apresentou média de otimismo 22,38 \pm 3,61 (Neves, 2015). Esta comparação, ainda que frágil pela diferença das características da amostra, indica que a amostra do presente estudo apresentou pontuação geral mais alta e menor variabilidade de otimismo.

Sobre as distorções cognitivas, a pontuação do $\mathrm{CD}$-Quest individual variava entre 0 a 5 , e aquelas que tiveram maior média foram "afirmações do tipo deveria" (média $1,7 \pm 1,5$ ), seguida de "pensamento dicotômico" $(1,5 \pm 1,4)$. A pontuação total do CD-Quest, ou seja, a soma de todos os escores de distorção cognitiva, apresentou média 16,6 $\pm 13,1$. Como se trata de um instrumento novo, poucas pesquisas foram encontradas utilizando este questionário. Um estudo brasileiro sobre distorções cognitivas em pessoas com obesidade mórbida (Da Luz, 2013) teve média total de distorções cognitivas de 18,36 $\pm 15,02$, sendo as distorções com maior média as "afirmações do tipo deveria" $(2 \pm 1,8)$, seguida de "raciocínio emocional" $(1,8 \pm 1,7)$. A pesquisa de validação brasileira do CD-Quest obteve média total de distorções cognitivas de 22,59 $\pm 11,86$, sendo as distorções com maior média as "afirmações do tipo deveria" $(2,3 \pm 1,5)$, seguida de "questionamentos e se" $(2,2 \pm 1,5)$ (Carvalho, 2014). Apesar de se tratar de amostras com perfis distintos do atual estudo, este apresentou menor escore de distorções cognitivas e variabilidade intermediária. Nota-se que todos apresentaram como distorção mais comum as "afirmações do tipo deveria", que são definidas no instrumento como a tendência a dizer que os acontecimentos, comportamentos e atitudes de si e dos outros deveriam ser da maneira que o indivíduo espera que sejam (Carvalho, 2014). Tal prevalência desta distorção também foi encontrada na pesquisa de validação deste instrumento na Turquia (Batmaz, Kocbiyik \& Yuncu, 2015), ficando em segundo lugar em sua pesquisa de validação na Austrália (Kostoglou \& Pidgeon, 2015). Apesar da escassez de bases para comparação, os dados indicam que as distorções encontradas na 
presente pesquisa são comuns a outros estudos, no Brasil e no mundo.

Acerca da diferença entre os grupos, no início do estudo foram levantadas hipóteses com base na literatura específica sobre o tema da HA e o estresse. A primeira hipótese foi a de que a amostra de pacientes com HA estaria em fases mais avançadas de estresse e teria maior escore de distorções cognitivas, além de menores índices de otimismo do que as pessoas sem HA.

Quanto à correlação entre estresse e HA levantada na hipótese, existem referências sobre a associação destas variáveis em múltiplos estudos (Malagris et al., 2014; Treiber et al., 2003). Na presente pesquisa, entretanto, não houve diferença significativa entre os grupos quanto ao número de pessoas estressadas ( $p=0,288)$, número de sintomas ( $p=0,183)$, tipos de sintomas $(p=0,365)$ ou fases do estresse $(p=0,636)$. Como esta hipótese não foi confirmada, algumas investigações foram feitas acerca da amostra. Uma vez que este estudo, em especial, contou com grande presença de militares e ex-militares (todos os participantes do sexo masculino), decidiu-se verificar a possibilidade desta composição ter interferido na vulnerabilidade ao estresse. As particularidades desta carreira incluem aspectos que se associam à redução do estresse, como a atividade física regular aeróbica (Rodrigues, Martinez, Duarte, \& Ribeiro, 2007), apoio social fornecido pela instituição e, em alguns contextos, a exposição a situações adversas (Cotian, Vilete, Volchan, \& Figueira, 2014). Mesmo excluindo os homens e, consequentemente, a parte da amostra composta de militares, não houve diferença significativa na proporção de pessoas com estresse entre os grupos ( $p=0,877$ ). Cabe ressaltar que, como a maior parte da amostra foi composta de militares, sua exclusão deixou esta investigação comprometida pela pequena quantidade de indivíduos $(\mathrm{n}=33)$.

Outra possível explicação para o estresse não ter se relacionado à HA seria o alto grau de otimismo encontrado no Grupo A, uma vez que há relatos de que esta variável pode funcionar como um fator de proteção ao estresse (Puig-Perez et al., 2015; Scheier \& Carver, 1992). Nesta pesquisa, cuja escala medida pelo LOT-R varia entre 0 e 30 pontos, a amostra total teve média $26,2 \pm 4,2$, havendo diferença significativa entre o Grupo A (média 27,36 $\pm 3,87$ ) e o Grupo B (média $25,22 \pm 4,37)(\mathrm{p}=0,017)$. Isto indica que as pessoas com HA relataram mais otimismo que as pessoas sem HA, o que será discutido posteriormente. Quanto à articulação entre estresse e otimismo, houve relação significativa e inversa entre a presença de estresse e o grau de otimismo no Grupo B ( $\mathrm{p}=0,008)$. Entretanto no Grupo A essa relação não foi encontrada ( $p=0899)$, sugerindo que, apesar dos escores de otimismo terem sido maiores nas pessoas com HA, isto não agiu como um fator de proteção ao estresse neste grupo.

Outra conjectura acerca do estresse não ter se manifestado de maneira significativamente diferente entre os grupos diz respeito ao fato de que os participantes já teriam recebido um tratamento por meio de equipe multiprofissional, composto de encontros regulares com nutricionistas, médicos e enfermeiros. Este tratamento, embora não vise o controle do estresse em si, é orientado a mudanças no estilo de vida, como reeducação alimentar e prática de atividades físicas, e supõe-se que possa ter contribuído para uma redução dos escores de estresse no Grupo A, aproximando seus valores em comparação ao Grupo B. Afinal, um dos critérios de inclusão para participação na pesquisa foi que o usuário tivesse diagnóstico de HA estabelecido há pelo menos um ano, e é prática da clínica médica da instituição promover o pronto encaminhamento para tratamento multiprofissional uma vez confirmado o diagnóstico. Cabe aqui destacar que o Treino de Controle de Stress Lipp possui como dois de seus quatro pilares a reeducação alimentar e o exercício físico regular (Lipp \& Malagris, 2011).

Outra hipótese é de que o alto otimismo no Grupo A seja anterior ao surgimento da HA e possa ter contribuído para seu aparecimento. A literatura indica que o otimismo em caráter exagerado está relacionado a comportamentos de risco mais frequentes (ReyesVelázquez \& Sealey-Potts, 2015). Acredita-se, portanto, que este otimismo, caso já existente antes do surgimento de HA, pode ter contribuído para maior exposição a comportamentos que levam à HA, como alimentação desbalanceada ou falta de acompanhamento médico. Estes comportamentos podem ser atribuídos a pessoas que acreditam que nada dará errado e que não é necessária a prevenção, ou que simplesmente não pensem nas consequências de comportamentos não saudáveis a longo prazo. Neste sentido, pode-se refletir sobre que um grau de otimismo moderado, ligado à realidade, tende a ser mais saudável do que o otimismo irreal (Reyes-Velázquez \& Sealey-Potts, 2015).

A hipótese inicial levantada também se refere à possível correlação entre HA, distorções cognitivas e otimismo. Apesar de não ter sido encontrado histórico 
na literatura deste tipo de investigação multivariada, há estudos indicando que, durante o tratamento de doenças crônicas, a atenção tende a se dirigir a consequências negativas (Evers et al., 2001). Supôs-se, portanto, que o maior número de restrições envolvidas no tratamento da HÁ e a maior chance de adquirir condições debilitantes poderiam favorecer o aparecimento de distorções cognitivas e inibir o otimismo. Sobre as distorções cognitivas, a pontuação total do CD-Questou seja, a soma de todos os escores de distorção cognitiva - apresentou média 16,6 $\pm 13,1$. Não houve diferença significativa entre as médias gerais do Grupo A $(16,04 \pm 11,95)$ e do Grupo B $(17,92 \pm 14,28)(p=0,97)$, bem como entre as médias de cada distorção, indicando que a presença de HA não se relacionou a uma maior ou menor pontuação de distorção cognitiva. Já a média de escores e o grau do otimismo, como já apresentado, foram maiores no grupo de pessoas com HA ( $p=0,017)$, o que chamou atenção por ter contrariado uma das hipóteses iniciais.

Conjectura-se que o tratamento com equipe multiprofissional, já exposto previamente, possa ter contribuído para o aumento de otimismo no Grupo A. Esta explicação carece de embasamento, uma vez que não houve um grupo controle em que pessoas com HA não estivessem em tratamento e não foi encontrado na literatura este tipo específico de investigação. Apesar do tema da promoção de otimismo ser recente e seus dados serem ainda escassos, alguns estudos apontam haver intervenções efetivas que atuam no aumento de pontuações de otimismo (Jenaabadi, 2014).

A segunda hipótese do estudo foi a de que existiria uma relação direta entre estresse e distorções cognitivas em pacientes com e sem HA, uma vez que a literatura já associa pessoas que distorcem mais a realidade a reações de estresse mais intensas (Rangé \& Masci, 2001). Esta correlação foi encontrada tanto na amostra geral quanto no Grupo A. Ainda que não se possa estabelecer relação de causa e consequência entre as variáveis, as pessoas estressadas tiveram significativamente maior média dos escores de distorções cognitivas do que as sem estresse. Um dado original desta pesquisa está no fato de que esta relação entre estresse e distorções cognitivas foi encontrada de maneira mais forte no Grupo A $(p=0,006)$, em comparação à amostra geral $(p=0,021), e$ não foi encontrada no Grupo $B(p=0,418)$. Na presença de HA, portanto, a relação entre estresse e distorção cognitiva pareceu se potencializar, enquanto em sua ausência esta relação se enfraqueceu a ponto de não ser significativa. A literatura demonstra que indivíduos diferentes podem ter reações brandas ou de estresse intenso frente a uma mesma situação (Vieira \& Lima, 2007), e que reações mais intensas podem se relacionar ao surgimento futuro de HA (Crump, Sundquist, Winkleby, \& Sundquist, 2016). Segundo Radulescu, Stoicescu, Buzdugan e Donca (2013), em pessoas predispostas isto pode ocorrer devido à hipertrofia do músculo cardíaco por meio de um processo de adaptação gerado pelos frequentes aumentos de PA, eliciados por reações mais intensas ao estresse. Em consonância a estas ideias, o presente estudo conjecturou que as distorções cognitivas podem eliciar maior resposta de estresse em determinadas pessoas, que, por conta desta variável somada a outras, podem vir a apresentar HA. Desta forma, tenderiam a registrar maior nível de estresse em comparação a pessoas sem HA, o que explicaria o fato da relação entre estresse e distorções cognitivas ser encontrada significativamente no Grupo A, e não no $B$.

A terceira hipótese do presente estudo era de que a relação entre as medidas de distorção cognitiva e estresse seria significativa nos pacientes com HA, e não nos indivíduos sem HA. Esta hipótese foi formulada a partir de estudos que apontam que uma das etiologias para o surgimento da HA seria a alta reatividade cardiovascular a situações de estresse (Treiber et al., 2003). Havia a expectativa de que distorções cognitivas pudessem estar relacionadas a reações mais intensas de estresse em pessoas com HA em comparação com pessoas sem HA. A hipótese foi confirmada, pois, como já apresentado, a relação entre o estresse e as distorções cognitivas mostrou-se diferente entre os grupos, sendo significativa e direta no Grupo A e inexistente no Grupo B. Os dados indicam, ainda, que algumas distorções analisadas individualmente associaram-se significativamente ao estresse, apresentando diferença entre os grupos acerca dos tipos.

Considerando as distorções isoladamente em relação ao estresse, apenas a distorção "ampliação/ minimização" relacionou-se significativamente à presença de estresse no Grupo A $(p=0,04)$. Isto indica que os indivíduos do Grupo A que mais apresentaram "ampliação/minimização" tiveram maiores escores de estresse do que os que tiveram outros tipos de distorção. Conjectura-se que, no caso de pessoas com HA, o componente "ampliação" desse grupo de distorções possa ter especial importância no nível de estresse, uma vez que pode estar relacionado a avaliações exageradas de seus sintomas, interpretando riscos que podem aumentar 
seu nível de estresse. É difícil afirmar se, ao responder o instrumento sobre distorções, no caso específico da "ampliação/minimização", a pessoa estaria se referindo à ampliação ou minimização, ou, ainda, a ambas concomitantemente. No presente estudo não foram explorados quais conteúdos fizeram parte das distorções, não sendo possível, inclusive, concluir se foram ligados à saúde ou a outras questões, ou mesmo se surgiram antes ou depois do aparecimento da HA. Já no Grupo B, apenas a "previsão do futuro" ( $p=0,01)$, "leitura mental" $(p=0,03)$ e os "questionamentos e se" $(p=0,02)$, relacionaram-se significativamente à presença de estresse.

Cabe enfatizar que não houve diferença significativa entre os grupos acerca de qualquer distorção. Os grupos não diferiram na proporção de pessoas que tiveram distorções, mas sim na relação entre as distorções e o estresse. Ainda que estes dados necessitem de maiores investigações, apontam no sentido de que as pessoas com e sem HA podem ter vulnerabilidades distintas ao estresse, e o tratamento orientado a estas diferenças pode trazer maiores benefícios. Importante ressaltar que estas associações não permitem a conclusão entre causas e consequências, não sendo possível estabelecer se são as distorções que elevariam o estresse, se o estresse aumentaria a proporção das distorções ou se há outro fator interferindo em ambos. Vale lembrar, ainda, que os grupos diferiram em termos de otimismo, o que pode ter influenciado nestes resultados.

A última hipótese deste estudo era de que haveria uma relação inversa entre estresse e otimismo em pacientes com e sem HA. Esta hipótese foi formulada a partir de estudos indicando que o otimismo pode funcionar como um fator de proteção ao estresse (Puig-Perez et al., 2015; Scheier \& Carver, 1992). Como já apresentado, esta relação foi encontrada no Grupo B de maneira inversa, mas não no Grupo A.

É possível que em pessoas com doenças crônicas o papel do otimismo como fator de proteção ao estresse funcione de maneira limitada, como sugerido pelo trabalho de Puig-Perez, Hackett, Salvador e Steptoe (2017), em que as pessoas mais otimistas demonstraram medidas mais saudáveis de PA em resposta ao estresse, mas não menor medida de cortisol ao acordar, que é uma mensuração muito associada à percepção de estresse. Os próprios autores discutem a possibilidade de a doença crônica ter interferido nesta medida, uma vez que outros estudos, sem envolver doentes crônicos, demonstram que a resposta de cortisol ao acordar acompanha as demais mensurações de estresse. Entretanto, não formularam hipóteses que explicassem esta diferença em pessoas com doenças crônicas (Puig-Perez et al., 2017). No presente estudo, especificamente quanto à $\mathrm{HA}$, conjecturou-se a possibilidade de as pessoas com HA terem maior sensibilidade ao estresse, ou seja, terem respostas de estresse mais elevadas que pessoas sem HA, de modo que cognições otimistas possam não ter força suficiente para agir como um fator de proteção significativo.

No Grupo B, a distorção do tipo "questionamentos e se" agiu de maneira moderadora, interferindo de maneira significativa na relação entre otimismo e estresse. Isto é, as pessoas que relataram esta distorção apresentaram relação inversa entre otimismo e estresse menor $(p=0,148)$ em comparação aos que não a apresentaram $(p<0,001)$. Segundo De Oliveira et al. (2015), esta distorção é caracterizada pelo contínuo questionamento sobre as situações acontecerem de forma negativa ("e se meu carro bater?"; "e se meu casamento acabar?"). Não foi encontrada na literatura esta relação, mas uma possível explicação para este efeito moderador diz respeito ao conteúdo destes questionamentos realizados pelo indivíduo, que podem ir de encontro aos pensamentos otimistas. Conjectura-se, deste modo, que ao ter pensamentos positivos o sujeito que tende a este tipo de distorção adicione novos questionamentos negativos, mantendo o nível de estresse alto, ainda que tenha havido uma cognição otimista em algum momento.

Os resultados indicam que a presença de HA, portanto, pode interferir na relação entre otimismo e estresse. Afinal, ainda que moderado por uma distorção cognitiva, o otimismo agiu como um fator de proteção ao estresse no grupo sem HA, o que não ocorreu no grupo com HA.

\section{Considerações finais}

Este estudo teve o objetivo de comparar um grupo de pessoas com HA com outro sem HA em suas relações com o estresse, otimismo e distorções cognitivas. Os resultados apontaram diferenças nas relações entre essas três variáveis ao comparar os dois grupos. Mais especificamente, naquele com ausência de HA houve relação inversa entre estresse e otimismo, moderada pela distorção do tipo "questionamentos e se", enquanto naquele com presença de HA houve relação direta apenas entre distorções cognitivas e estresse. Portanto, ainda que sejam necessárias investigações mais aprofundadas e não seja possível a afirmação categórica, os 
resultados indicam a possibilidade de que a presença de HA, em meio a outras variáveis, possa enfraquecer a relação inversa entre estresse e otimismo, enquanto fortalece a relação direta entre estresse e distorções cognitivas.

Outro resultado interessante foi o fato de que, mesmo que a HA não tenha se relacionado a qualquer tipo específico de distorção cognitiva, houve diferenças entre os tipos específicos de distorção significativamente associados ao estresse dentro dos grupos. Este dado indica a possibilidade de que pessoas com HA podem ter maior sensibilidade a gerar estresse ao distorcer a realidade de modo a "ampliar/minimizar" do que pessoas sem HA. Ou, ainda, que pessoas com HA podem ter maior tendência a "ampliar/minimizar" quando estão mais estressadas.

Em relação às limitações do estudo, pode-se afirmar que não foi realizado um controle rigoroso da variável idade, o que fez que o grupo com HA (Grupo A) tivesse média significativamente maior do que o B. Isso pode ter interferido nos resultados de comparação entre os grupos. Além disto, todos os participantes com HA estavam em tratamento multiprofissional, ainda que sem intervenções psicológicas. Este tratamento pode ter agido sobre o otimismo, e seria interessante explorar em grupos controlados se diferentes intervenções, ainda que não psicológicas, podem atuar sobre esta variável. Sobre os instrumentos utilizados, cabe apontar que os estudos de validação do LOT-R e o CD-Quest foram realizados com indivíduos mais jovens do que os da amostra utilizada pela presente pesquisa, o que pode ter comprometido a precisão de seus resultados. Por fim, algumas comparações de categorias foram limitadas pela quantidade de participantes, e sabe-se que quanto maior a amostra mais confiáveis são os apontamentos encontrados.

Apesar das limitações, os resultados se revelam interessantes e relevantes no entendimento das relações estudadas, podendo servir de base para futuros estudos que procurem manejar as limitações e que envolvam um número maior de participantes.

\section{Referências}

Bastianello, M., \& Pacico, J. (2014). Otimismo. In R. Hutz (Org.), Avaliação em psicologia positiva (pp. 95-100). Porto Alegre, RS: Artmed.

Batmaz, S., Kocbiyik, S., \& Yuncu, O. (2015). Turkish version of the cognitive distortions questionnaire: psychometric properties. Depression Research and Treatment, 2015, 1-8. http://doi.org/10.1155/2015/694853

Beck, J. (2013). Terapia cognitivo-comportamental. São Paulo, SP: Artmed.

Blom, K., Baker, B., How, M., Dai, M., Irvine, J., Abbey, S., Abramson, B. L., Myers, M. G., Kiss, A., Perkins N. J., Tobe, S. W. (2014). Hypertension analysis of stress reduction using mindfulness meditation and yoga: results from the harmony randomized controlled trial. American Journal of Hypertension, 27(1), 122-129. http://dx.doi.org/10.1093/ajh/hpt134

Brasil. (2014). Vigitel Brasil 2013: vigilância de fatores de risco e proteção para doenças crônicas por inquérito telefônico. Brasília, DF: Ministério da Saúde. bvsms.saude.gov.br/bvs/publicacoes/vigitel_brasil_2014.pdf

Cade, N. (2001). Terapia de grupo para pacientes com hipertensão arterial. Revista de Psiquiatria Clínica, 28(6), 300-341.

Carvalho, C. (2014). Análise psicométrica do questionário de distorções cognitivas (Tese de doutorado). Universidade Federal da Bahia, Salvador, BA.

Carver, C., Scheier, M., \& Segerstrom, S. (2010). Optimism. Clinical Psychology Review, 30, 879-889. http://dx.doi.org/ 10.1016/j.cpr.2010.01.006

Chang, Y., \& Chan, H. (2015). Optimism and proactive coping in relation to burnout among nurses. Journal of Nursing Management, 23(3), 401-408. https://doi.org/10.1111/jonm.12148

Cotian, M. S., Vilete, L., Volchan, E., \& Figueira, I. (2014). Revisão sistemática dos aspectos psicossociais, neurobiológicos, preditores e promotores de resiliência em militares. Jornal Brasileiro de Psiquiatria, 63(1), 72-85. http://doi.org/10.1590/0047-2085000000009

Costa, M., Accioly, H., Jr., Oliveira, J., \& Maia, E. (2007). Estresse: diagnóstico dos policiais militares em uma cidade brasileira. Revista Panamericana de Salud Publica, 21(4), 217-222. https://doi.org/10.1590/s102049892007000300004

Crump, C., Sundquist, J., Winkleby, M., \& Sundquist, K. (2016). Low stress resilience in late adolescence and risk of hypertension in adulthood. Heart, 102(7), 541-547. http://dx.doi.org/10.1136/heartjnl-2015-308597 
Da Luz, F. (2013). Distorções cognitivas, esquemas iniciais desadaptativos, depressão, ansiedade e estresse em obesos mórbidos e pessoas com peso normal (Tese de doutorado). Universidade Católica do Rio Grande do Sul, Porto Alegre, RS.

Dantas, M., Brito, D., Rodrigues, P., \& Maciente, T. (2010). Avaliação de estresse em policiais militares. Psicologia, Teoria e Prática, 12(3), 66-77. http://pepsic.bvsalud.org/scielo.php?script=sci_arttext\& pid=S1516-36872010000300006\&lng=pt\&nrm=iso\&tlng=pt

Evers, A., Kraaimaat, F, Van Lankveld, W., Jongen, P., Jacobs, J., \& Bijlsma, J. (2001). Beyond unfavorable thinking: the illness cognition questionnaire for chronic diseases. Journal of Consulting and Clinical Psychology, 69, 10261036. https:// doi.org/10.1037//0022-006x.69.6.1026

Jose, R., \& D'Almeida, V. (2013). Effectiveness of Jacobson's progressive muscle relaxation (JPMR) on blood pressure and health related stress level among patients with hypertension in a selected hospital of Mangalore. International Journal of Nursing Education, 5(2), 171-175. https://doi.org/10.5958/j.0974-9357.5.2.089

Gustafsson, H., \& Skoog, T. (2012). The mediational role of perceived stress in the relation between optimism and burnout in competitive athletes. Anxiety, Stress \& Coping: An International Journal, 25(2), 183-199. https://doi.org/ $10.1080 / 10615806.2011 .594045$

Instituto Brasileiro de Geografia e Estatística. (2012). Censo demográfico 2010. Rio de Janeiro, RJ: IBGE. https://biblioteca.ibge.gov.br/visualizacao/periodicos/94/cd_2010_religiao_deficiencia.pdf

Jenaabadi, H. (2014). The effect of training skills of optimism on fostering emotional intelligence of males in Education \& Improvement center in Zahedan. Social and Behavioral Sciences, 114, 191-196. https://doi.org/10.1016/ j.sbspro.2013.12.683

Kostoglou, S., \& Pidgeon, A. (2016). The cognitive distortions questionnaire: psychometric validation for an australian population. Australian Journal of Psychology, 68(2), 123-129.

Lipp, M. (2000). Inventário de sintomas de stress para adultos de lipp. São Paulo, SP: Casa do Psicólogo.

Lipp, M., \& Malagris, L. (2011). Estresse: aspectos históricos, teóricos e clínicos. In B. Rangé (Org), Psicoterapias cognitivo-comportamentais: um diálogo com a psiquiatria (pp. 617-632). Porto Alegre, RS: Artmed.

Malagris, L., Lipp, M., \& Chicayban, L. (2014). Hipertensão arterial sistêmica: contribuição de fatores emocionais e possibilidades de atuação da psicologia. In E. Seidl \& M. Miyazaki, Psicologia da saúde: pesquisa e atuação profissional no contexto de enfermidades crônicas (pp. 73-102). São Paulo, SP: Juruá.

Malagris, L., Brunini, T., Moss, M., Silva, P., Esposito, B., \& Ribeiro, A. (2009). Evidências biológicas do treino de controle do stress em pacientes com hipertensão. Psicologia: Reflexão e Crítica, 22(1), 60-68. https://doi.org/10.1590/ s0102-79722009000100009

Margis, R. (2003). Comorbidades no transtorno de estresse pós-traumático: regra ou exceção? Revista Brasileira de Psiquiatria, 25, 17-20. https://doi.org/10.1590/s1516-44462003000500005

Neves, S. (2015). Satisfação com a vida em militares da Guarda Nacional Republicana do Comando Territorial de Leiria: relação com a afetividade positiva e negativa, pessimismo e otimismo (Dissertação de mestrado). Universidade Lusófona de Humanidades e Tecnologias, Lisboa.

Oliveira, I., Seixas, C., Osório, F., Cripa, J., Abreu, J., Menezes, I., Pidgeon, A., Sudak, D., \& Wenzel, A. (2015). Evaluation of the psychometric properties of the cognitive distortions questionnaire (CD-Quest) in a sample of undergraduate students. Innovations in Clinical. Neuroscience, 12(7-8), 20-27. https://www.ncbi.nlm.nih.gov/pmc/ articles/PMC4558787

Oliveira-Martins, S., Oliveira, T., Gomes, J., Caramona, M., \& Cabrita, J. (2011). Factores associados à hipertensão arterial nos utentes de farmácias em Portugal. Revista de Saúde Pública, 45(1), 136-144. https://doi.org/10.1590/ s0034-89102010005000056

Oliveira, P., \& Bardagi, M. (2009). Estresse e comprometimento com a carreira em policiais militares. Boletim de Psicologia, 59(131), 153-166. http:/ / pepsic.bvsalud.org/scielo.php?script=sci_arttext\&pid=S0006-59432009000200003

Puig-Perez, S., Hackett, R., Salvador, A., \& Steptoe, A. (2017). Optimism moderates psychophysiological responses to stress in older people with Type 2 diabetes. Psychophysiology, 54(4), 536-543. https://doi.org/10.1111/psyp.12806

Puig-Perez, S., Villada, C., Pulopulos, M., Almela, M., Hidalgo, V., \& Salvador, A. (2015). Optimism and pessimism are related to different components of the stress response in healthy older people. International Journal of Psychophysiology, 98, 213-221. https://doi.org/10.1016/j.ijpsycho.2015.09.002 
Radulescu, D., Stoicescu, L., Buzdugan, E., \& Donca, V. (2013). Patterns of left ventricular remodeling among patients with essential and secondary hypertension. Revista Medica de Chile, 141(12), 1520-1527. https:// doi.org/10.4067/s0034-98872013001200004

Rangé, B., \& Masci, C. (2001). Transtorno de estresse pós-traumático. In B. Rangé (Org.), Psicoterapias cognitivo-comportamentais: um diálogo com a psiquiatria (pp. 257-274). Porto Alegre, RS: Artmed.

Reyes-Velázquez, W., \& Sealey-Potts, C. (2015). Unrealistic optimism, sex, and risk perception of type 2 diabetes onset: implications for education programs. Diabetes Spectrum, 28(1), 5-9. https://doi.org/10.2337/diaspect.28.1.5

Rodrigues, A., Martinez, E., Duarte, A., \& Ribeiro, L. (2007). O condicionamento aeróbico e sua influência na resposta ao estresse mental em oficiais do Exército. Brasileira de Medicina do Esporte, 13(2), 113-117.

Scheier, M., \& Carver, C. (1992). Effects of optimism in psychological and physical well-being: theoretical overview and empirical update. Cognitive Therapy and Research, 16(2), 201-228. https://doi.org/10.1007/bf01173489

Schrank, B., Brownell, T., Tylee, A., \& Slade, A. (2014). Positive psychology: an approach to supporting recovery in mental illness. East Asian Arch Psychiatry, 24, 95-10. http://www.easap.asia/index.php/component/k2/item/ 248-1403-v24n3-p953

Seligman, M. (2011). Florescer. Rio de Janeiro, RJ: Objetiva.

Serra, M., Pereira, L., Fontenele, D., Viveiros, M., \& Lima, R. (2015). Condições clínicas e antropométricas de hipertensos atendidos em um centro de saúde de São Luís. Revista Pesquisa e Saúde, 16(2), 107-111. www.periodicoseletronicos.ufma.br/index.php/revistahuufma/article/view/4241/2272

Sociedade Brasileira de Cardiologia, Sociedade Brasileira de Hipertensão, \& Sociedade Brasileira de Nefrologia. (2010). VI Diretrizes brasileiras de hipertensão arterial. Arquivos Brasileiros de Cardiologia, 95(1), 1-51. http:/publicacoes.cardiol.br/consenso/2010/Diretriz_hipertensao_associados.pdf

Thomas, J., Britt, T., Odle-Dusseauet, H., \& Bliese, P. (2011). Dispositional optimism buffers combat veterans from the negative effects of warzone stress on mental health symptoms and work impairment. Journal of Clinical Psychology, 67(9), 866-880. https://doi.org/10.1002/jclp.20809

Treiber, F, Kamarck, T., Schneiderman, N,. Sheffield, D., Kapuku, G., \& Taylor, T. (2003). Cardiovascular reactivity and development of preclinical and clinical disease states. Psychosomatic Medicine, 65 (1), 46-62. https://doi.org/10.1097/00006842-200301000-00007

Vieira, F., \& Lima, E. (2007). Testes de estresse laboratoriais e hipertensão arterial. Revista Brasileira de Hipertensão, 14(2), 98-103. http://www.scielo.mec.pt/pdf/psd/v5n2/v5n2a07.pdf

Diego dos Santos Gonçalves Pacheco

Mestre em Psicologia pela Universidade Federal do Rio de Janeiro, Rio de Janeiro - RJ. Brasil.

E-mail: diegoprj@gmail.com

(1) https://orcid.org/0000-0003-0814-5899

\section{Lucia Emmanoel Novaes Malagris}

Mestre em Psicologia Clínica pela Pontífica Universidade Católica de Campinas, Campinas - SP, Brasil. Doutora em Fisiopatologia Clínica e Experimental pela Universidade do Estado do Rio de Janeiro, Rio de Janeiro - RJ. Brasil.

E-mail: lucia.novaes.malagris@hotmail.com

(1) https://orcid.org/0000-0001-9822-5467

Endereço para envio de correspondência:

Policlínica Naval de Campo Grande, Departamento de Saúde, Seção de Psicologia. Av. Brasil, 44.878, Campo

Grande. CEP: 23078-001. Rio de Janeiro - RJ. Brasil. 
Psicologia: Ciência e Profissão 2020 v. 40, e186605, 1-12.

Recebido 17/10/2017

Aceito 03/03/2020

Received 17/10/2017

Approved 03/03/2020

Recibido 17/10/2017

Aceptado 03/03/2020

Como citar: Pacheco, D. S. G., \& Malagris, L. E. N. (2020). Relações entre Estresse, Distorções Cognitivas e Otimismo em Pacientes com Hipertensão. Psicologia: Ciência e Profissão, 40, 1-12. https://doi.org/10.1590/1982-3703003186605

How to cite: Pacheco, D. S. G., \& Malagris, L. E. N. (2020). Relations between Stress, Cognitive Distortions and Optimism in Patients with Hypertension. Psicologia: Ciência e Profissão, 40, 1-12. https://doi.org/10.1590/1982-3703003186605

Cómo citar: Pacheco, D. S. G., \& Malagris, L. E. N. (2020). Relaciones entre Estrés, Distorsiones Cognitivas y Optimismo en Pacientes con Hipertensión. Psicologia: Ciência e Profissão, 40, 1-12. https://doi.org/10.1590/1982-3703003186605 